\title{
Analysis of Temperature Effects on Raman Silicon Photonic Devices
}

\author{
Wonchul Kim and Dongwook Park* \\ School of Electronic and Electrical Engineering, Hongik University, Seoul, 121-791, Korea
}

(Received October 8, 2008 : revised November 13, 2008 : accepted November 13, 2008)

\begin{abstract}
Recent research efforts on study of silicon photonics utilizing stimulated Raman scattering have largely overlooked temperature effects. In this paper, we incorporated the temperature dependences into the key parameters governing wave propagation in silicon waveguides with Raman gain and investigated how the temperature affects the solution of the coupled-mode equations. We then carried out, as one particular application example, a numerical analysis of the performance of wavelength converters based on stimulated Raman scattering at temperatures ranging from $298 \mathrm{~K}$ to $500 \mathrm{~K}$. The analysis predicted, among other things, that the wavelength conversion efficiency could decrease by as much as $12 \mathrm{~dB}$ at $500 \mathrm{~K}$ in comparison to that at the room temperature. These results indicate that it is necessary to take a careful account of temperature effects in designing, fabricating, and operating Raman silicon photonic devices.
\end{abstract}

Keywords: Temperature dependence, Stimulated raman scattering, Silicon photonics, Raman susceptibility, Raman gain coefficient, Parametric wavelength conversion, Coupled-mode equations, Coupling coefficients.

OCIS codes : (130.4310) Nonlinear; (160.4760) Optical properties; (190.4970) Parametric oscillators and amplifiers; (190.5650) Raman effect; (290.5910) Scattering, stimulated Raman

\section{INTRODUCTION}

One of the main motivations in the recent drive behind the so-called silicon photonics is the potential of realizing cost-effective photonic components by using silicon, instead of compound semiconductors [1-3], as the platform. However, in order to benefit from the cost-reduction associated with silicon due to its simpler and more mature fabrication technologies as well as its integrability with other silicon-based electronic components, the problem of indirect-bandgap inherent in silicon must first be overcome. Optical gain due to stimulated Raman scattering (SRS) offers a possible solution in this regard as the Raman gain in silicon is several orders of magnitude greater than that of silica [4]. Currently, three areas are mainly being explored for potential applications of SRS-based silicon photonics: Raman amplifier [4], Raman lasers [4,5] and Raman parametric wavelength converters [4],[6].

Despite the recent interests, most research efforts on silicon photonics based on the SRS mechanism up to

*Corresponding author: dwpark@hongik.ac.kr this point have largely overlooked temperature effects on the device performance due to the complexity of the analysis involved. Analyses and measurements performed in the past on the topic have been confined mainly to characterization of bulk silicon and not photonic devices [7-11]. Some work have also been done pertaining to optical fiber $[12,13]$, but applicability of those results to silicon is severely limited because of the large discrepancies between the Raman characteristics of silicon and silica. The issue of temperature effects on Raman-based optical components is significant in two regards. One, the Raman scattering process is inherently sensitive to temperature variations, given the fact that the process involves a nonlinear phonon-photon interaction $[7,8]$ and the characteristics of phonons, which are quantized lattice vibrations, are highly-dependent on the temperature. Two, the temperature in devices utilizing Raman effects is expected to be quite high as high-intensity optical beams are usually required in such nonlinear applications. Clearly, the temperature effects must be carefully taken into account when designing and operating Raman-based devices. 
In this paper, we have incorporated the temperature dependence of key Raman parameters of bulk silicon, such as the Stokes and anti-Stokes frequency shift and the width of the resonant frequency response, into the parameters of the coupled-mode equations governing the wave propagation of the pump, Stokes and antiStokes components in a silicon waveguide structure with Raman gain. The coupled-mode equations were then solved numerically to yield the temperature dependence of a prototypical Raman parametric wavelength converter's efficiency, a result which we believe is being reported in the literature for the first time.

This paper is organized as follows. In Section II, we first present a brief background on stimulated Raman processes in silicon and on silicon rib waveguide, the guiding structure assumed in the paper. Next, an account of temperature-dependence models for the key Raman process parameters is given. Then, the coupled-mode equations that govern the propagation of the pump, Stokes and anti-Stokes beams in the waveguide with Raman gain are presented, wherein the temperature dependence of the Raman process is incorporated via the equations' coupling coefficients whose values depend directly on the aforementioned Raman parameters. In Section III, we demonstrate a specific application of our model by numerically solving the appropriate coupledmode equations and obtaining the conversion efficiency of a Raman wavelength converter for several different temperatures under various length and phase-matching conditions. The calculation results, among others, predict that the conversion efficiency can be degraded by as much as $12 \mathrm{~dB}$ due to a temperature increase of $200 \mathrm{~K}$, which suggests that temperature effects could pose a serious problem that must be dealt with in Raman-based silicon devices. The paper concludes with a brief summary and a closing remark in Section IV.

\section{BASIC CONCEPTS AND THEORY}

\section{A. Stimulated Raman scattering (SRS) and silicon rib waveguide}

Raman scattering is an inelastic nonlinear optical phenomenon wherein a photon and an optical phonon interact to generate a new photon at a shifted frequency commensurate with momentum and energy conversation laws; the up- and down-shifted frequency components are called the anti-Stokes and Stokes components, respectively $[14,15]$. If the pump beam is of sufficient intensity, a fairly efficient energy transfer among the pump, Stokes and anti-Stokes components can take place in silicon, providing the basis for SRS-assisted silicon photonic devices.

The physical origin of the optical phonon taking part in Raman scattering is lattice vibration, and thus the optical phonon's characteristics depend on the particular

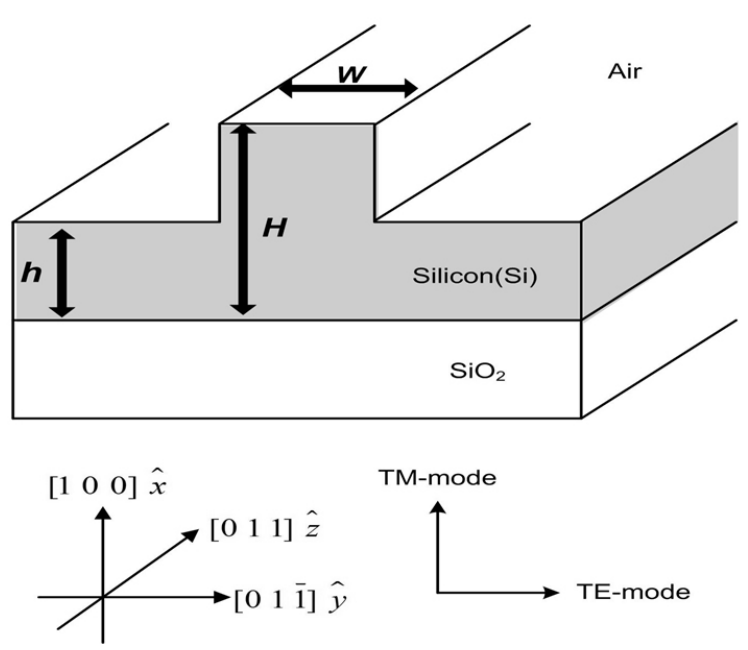

(a)

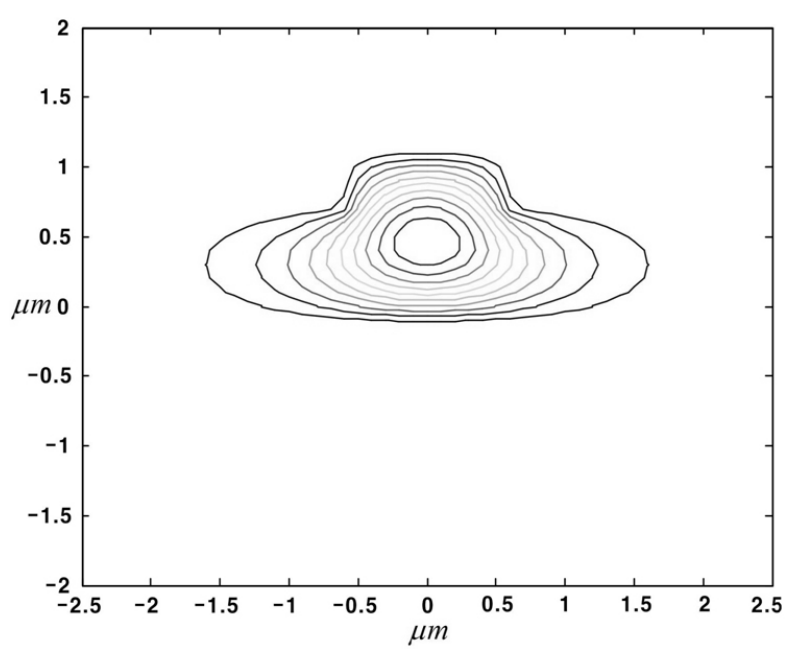

(b)

FIG. 1. Silicon rib waveguide assumed in the analysis: (a) Structure and orientation of the silicon rib waveguide ( $W=H=1 \mu \mathrm{m}, h=0.6 \mu \mathrm{m}$ ), (b) Fundamental mode profile of the waveguide at $\lambda=1434 \mathrm{~nm}$.

propagation direction along the crystal structure. In this paper, we shall assume that the silicon waveguide structure, the basic building block for various types of Raman photonic devices, is of the rib waveguide type directed along the direction (see Fig. 1(a)), a choice which is desirable in two respects. One, this orientation is opted for in most commercial CMOS silicon fabrication, which would render the existing facilities readily available for low-cost production of Raman photonic devices. Two, the nominal Raman shift in this direction is approximately $\pm 15.6 \mathrm{THz}[9],[16]$ at room temperature, making it suitable for wavelength conversion between the two commonlyused optical communication frequency bands, namely C- and O-bands.

Fig. 1(a) is a schematic of the silicon rib waveguide to be analyzed in this paper, wherein the waveguide 
direction and the TE/TM modes' orientations are indicated. The structure was chosen to be similar to that of $[6],[17]$ for the purpose of being able to cross-check the validity of our results. Fig. 1(b) is the fundamental mode's transverse profile for the structure. The latter was generated from application of a finite difference method based on a scalar wave assumption for $\lambda=$ $1434 \mathrm{~nm}$, with the material index value obtained from the Sellmeier equation for silicon [12]. The calculated effective core area and effective refractive index values are $A_{\text {eff }}=1.1349 \mu^{2}$ and $n_{\text {eff }}=3.4138$ respectively, These values are later used in the coupled-mode calculations in Section III.

\section{B. Temperature dependence of stimulated Raman scattering}

In order to investigate the effects of temperature change on photonic devices based on the Raman process, we first need to examine the temperature dependence of the optical phonon itself and also Raman parameters such as the Raman gain and susceptibility in silicon $[7,8],[10],[15]$.

The harmonic approximation for the potential function of the optical phonon, as given by the form $V=a x^{2}$, with $x$ being the displacement of the atomic particles from their equilibrium position, is valid only for those cases where $x$ is much less than the interatomic spacing, i.e., the lattice constant. However, when the magnitude of the displacement increases with temperature, as would happen in the case of Raman devices employing a high-intensity pump beam, higher-order terms of the potential function (the Morse-potential function is a commonly-used model [7]) need to be retained as well for a more accurate account of the temperature effects. Many of the recent theoretical results on Raman effects in silicon are based on the assumption of such a classical anharmonic oscillator model [7],[15]. Indeed these higherorder terms lead to a change in the nominal optical phonon frequency (Raman shift) as well as broadening of the phonon's spectral distribution $[7],[10],[15]$ as explained below.

From a phenomenological standpoint, a rise in temperature results in an increase in the interatomic distance and a decrease in the phonon lifetime [7],[10]. The former leads to a decrease in the optical phonon frequency - and a corresponding increase in the frequency of the Stokes component - while the latter, when interpreted as an increase in the damping coefficient in the context of a classical oscillator model, leads to broadening of the optical phonon spectrum, which also represents the resonance curve associated with the photon-phonon interaction in stimulated Raman scattering [7],[10]. Below we provide a short quantitative account of these effects.

The approximate temperature dependence of the lowestorder optical phonon's (resonance) frequency $v$ and the full-width-half-maximum (FWHM) $2 \Gamma$ of the optical phonon spectrum in silicon is given by the following expressions according to $[10],[18]$ :

$$
v(T)=v_{0}+\Delta v(T)
$$

where

$$
\Delta \nu(T)=A\left[1+\frac{2}{u^{1 / 2}-1}\right]+B\left[1+\frac{3}{u^{1 / 3}-1}+\frac{3}{\left(u^{1 / 3}-1\right)^{2}}\right]
$$

and

$$
2 \Gamma(T)=C\left[1+\frac{2}{u^{1 / 2}-1}\right]+D\left[1+\frac{3}{u^{1 / 3}-1}+\frac{3}{\left(u^{1 / 3}-1\right)^{2}}\right]
$$

with $A=-3.996 \times c, B=-0.235 \times c, C=1.683 \times c$, $D=0.1326 \times c, \nu_{0}=528 \times c$ and $u=\exp \left[h v_{0} / k_{B} T\right]$.

In the above, the units of $v(T), v_{0}, \Delta v(T)$ and $2 \Gamma(T)$,

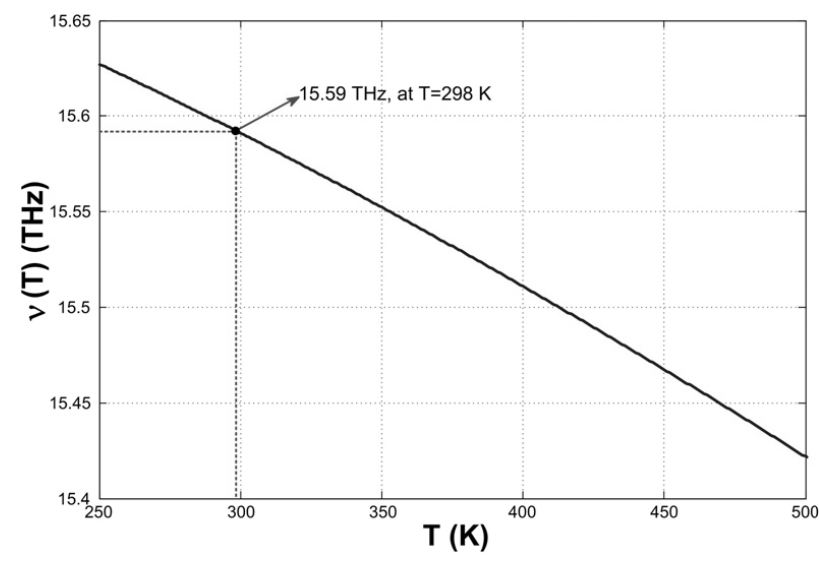

FIG. 2. Temperature dependence of the lowest-order resonance frequency of the optical phonon.

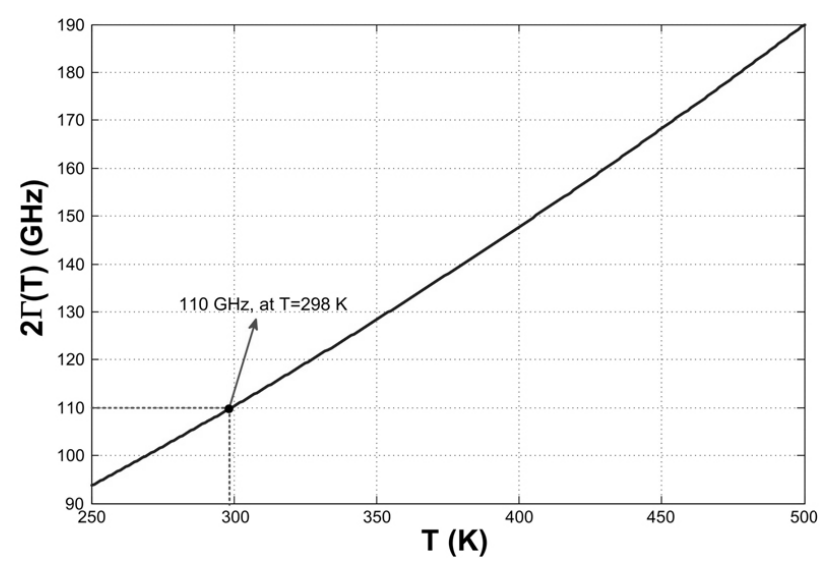

FIG. 3. Temperature dependence of the FWHM associated with the optical phonon spectrum. 
as well as those of $A, B, C$ and $D$, are all $\mathrm{Hz}$ and $c$ takes on the numerical value of $3 \times 10^{10}$. The symbols $h, k_{B}$ and $T$ represent the Planck's constant and Boltzmann's constant in SI units (with the lengths in $\mathrm{cm})$ - as are all quantities in subsequent discussions - and the absolute temperature in Kelvin $(K)$, respectively.

Figs. 2 and 3 depict the temperature dependence of $v$ and $2 \Gamma$ based on the above expressions and illustrate that the FWHM is much more sensitive to the temperature increase than the resonance frequency $v$. Whereas the resonance frequency decreases only by about 0.165 $\mathrm{THz}$, roughly $1 \%$ of the original value (corresponding to a change in the Stokes wavelength by about $1.4 \mathrm{~nm}$ for the case of the original Stokes wavelength equal to $1550 \mathrm{~nm}$ ) when the temperature is increased from 298 $\mathrm{K}$ to $500 \mathrm{~K}$, the FWHM is seen to increase by approximately $80 \mathrm{GHz}$ over the same temperature span, a dramatic increase of over $70 \%$. Such a large broadening of the optical phonon spectrum leads to a significant reduction in the Raman gain and Raman susceptibility as well as in the coupling coefficients of the coupled-mode equations, and ultimately a degradation of the efficiency of the Raman devices, as we shall see in the following discussions.

Raman susceptibility is an important parameter that characterizes the extent of interaction between an optical wave and the given Raman medium and plays a key role in the coupled-mode analysis as it directly determines the coupling coefficients. From a quantum mechanical analysis, the Raman susceptibility tensor for a signal (Stokes) beam of frequency $\nu_{S}$ in the presence of a pump beam of frequency can be expressed (in the unit of $\left.\mathrm{cm}^{2} / \mathrm{V}^{2}\right)$ in the following form [4],[19,20]:

$$
\chi_{i j m n}^{R}=2 \Gamma \nu \frac{n^{2} c g_{R}}{\pi \nu_{S} \eta_{0}} \frac{\sum_{k=1,2,3}\left(R_{i j}\right)_{k}\left(R_{m n}\right)_{k}}{\left(\nu^{2}-\left(\nu_{P}-\nu_{S}\right)^{2}+2 i \Gamma\left(\nu_{P}-\nu_{S}\right)\right)}
$$

In the above equation, $n$ is the medium's refractive index - taken to be $3.4-, c$ is the speed of light in vacuum, $3 \times 10^{10} \mathrm{~cm} / \mathrm{s}, \eta_{0}$ is the free-space intrinsic impedance, respectively, and $g_{R}$ is the on-resonance, i.e., the peak, Raman gain (see below for a discussion on this). $\left(R_{i j}\right)_{k}$ and $\left(R_{m n}\right)_{k}$ refer to the $i j$-th and $m n$-th components $(i, j, m, n=1,2,3)$ of the $k$-th Raman tensor $(k=$ $1,2,3)$, respectively, for the selected silicon waveguide direction ([ $\left[\begin{array}{lll}0 & 1 & 1\end{array}\right]$ in our case) and the choice of the axes [14],[16]. The Raman tensors are thus independent of the temperature. It must be remembered that the values of $2 \Gamma$ and $v$ to be used in the expression above are those corresponding to the actual temperature of the Raman medium. The expression clearly shows the effect of frequency detuning, namely the discrepancy between the optical phonon's resonance frequency $\nu$ at the prevailing temperature and $\nu_{P}-\nu_{S}$, the difference between the actual frequencies of the pump and the

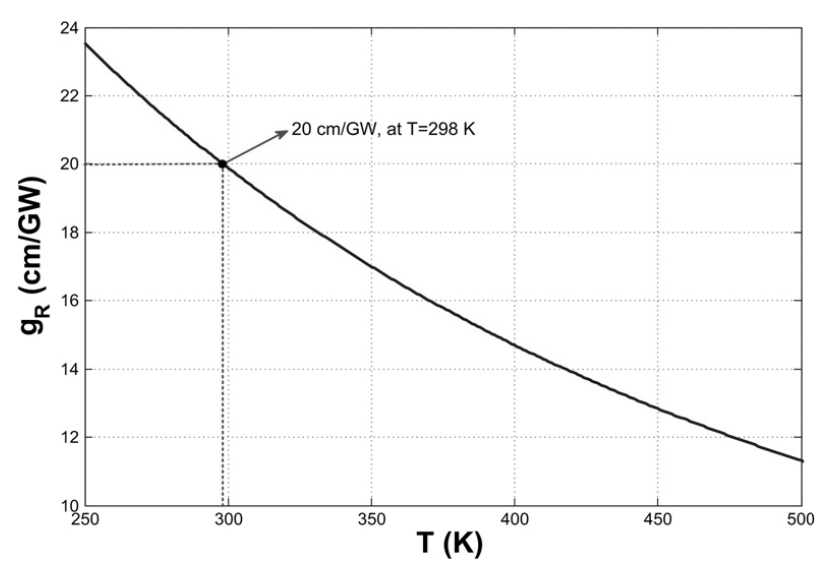

FIG. 4. Temperature dependence of the Raman gain coefficient at $\lambda_{S}=1550 \mathrm{~nm}$.

Stokes components which are injected into the medium.

The overall temperature dependence of the Raman susceptibility given by (4) is affected - in addition to being influenced directly through $v$ and $2 \Gamma$ as alluded to earlier - by that of the Raman gain coefficient $g_{R}$, a parameter characterizing the peak intensity gain, i.e., the gain at resonance or zero-detuning, experienced by the Stokes component (ignoring other effects such as the presence of the weak anti-Stokes component and intrinsic losses) in the following manner [9],[19-21]:

$$
g_{R}=\frac{2 c^{2}}{h \nu_{S}^{3} n^{2}(N+1) 2 \Gamma} S
$$

where $N$ is the Bose-Einstein occupation number $\left(N=\left[\exp \left(h v / k_{B} T\right)-1\right]^{-1}\right)$, and $S$ is the spontaneous Raman scattering efficiency. It has been reported that the Raman gain coefficient in silicon at $\lambda_{S}=1550 \mathrm{~nm}$ (with pump at $\lambda_{P}=1434 \mathrm{~nm}$ ) has been estimated to be $\sim 20 \mathrm{~cm} / \mathrm{GW}$ at room temperature $(298 \mathrm{~K})$ from Raman gain measurements [21]. As a side remark, we note that the Raman gain coefficient in silicon is much larger than that of silica $\left(0.93 \times 10^{-2} \mathrm{~cm} / \mathrm{GW}\right)$ at 1550 $\mathrm{nm}$ [4], primarily because of the difference in the value of FWHM $(2 \Gamma)$ for the two respective materials, $\sim 110$ $\mathrm{GHz}$ for silicon and $\sim 6 \mathrm{THz}$ for silica $[12]$ at room temperature. As a result, the one-way Raman gain for a device length of $1 \mathrm{~cm}$ and pump intensity of 0.1 $\mathrm{GW} / \mathrm{cm}^{2}$ - assuming $1 \mathrm{~W}$ pump beam power and $1 \mu \mathrm{m}^{2}$ effective core area for the sake of argument - would amount to $e^{2}$, a figure that would warrant serious considerations for building practical Raman amplifiers, lasers, and wavelength converters.

Fig. 4 illustrates the calculated overall temperature dependence of the Raman gain coefficient at $\lambda_{S}=1550$ $\mathrm{nm}$ based on (5), taking into account the temperature dependences of all the parameters involved, including the refractive index $n$ [22]. All of these parameters 


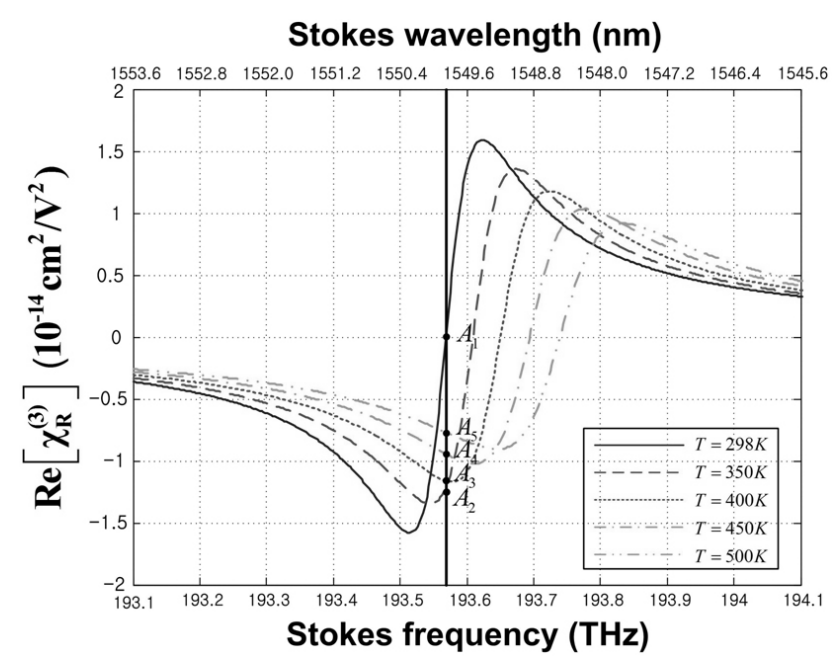

(a)

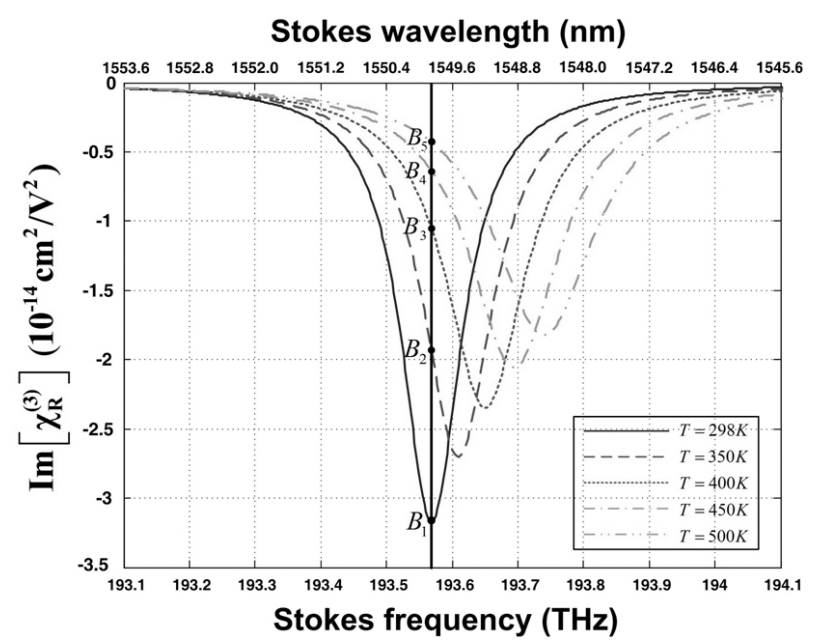

(b)

FIG. 5. Frequency detuning characteristics of the Raman susceptibility for various temperatures: (a) Real part, (b) Imaginary part.

change only slightly with temperature for the given range - calculated to be on the order of $10^{-2}$ order or less as the temperature is raised from $298 \mathrm{~K}$ to $500 \mathrm{~K}$ except for the FWHM, which, as alluded to earlier, increases by more than $70 \%$ over the same range. Thus, it is the change in the FWHM that dominates the temperature effect in the overall Raman gain coefficient and this observation is borne out by the fact that the Raman gain in Fig. 4 undergoes a decrease from $20 \mathrm{~cm} / \mathrm{GW}$ at $298 \mathrm{~K}$, a figure in very good agreement with the experimentally measured value [21], to a value less than $12 \mathrm{~cm} / \mathrm{GW}$, a reduction of $\sim 44 \%$. We shall now examine the temperature dependence of the Raman susceptibility based on (4) and the Raman gain result just obtained. Figs. 5(a) and (b) depict how, for those cases where $\chi_{i j m n}^{R}$ is nonzero (for example, $i j m n=1221,1212$, etc. for the $\left[\begin{array}{lll}0 & 1 & 1\end{array}\right]$ orientation of our waveguide [14],[16], for which the summation over the product of Raman tensor components in (4) reduces to unity), the real and imaginary parts of $\chi_{i j m n}^{R}$ - denoted simply by $\chi_{R}^{(3)}$ in the figures to symbolize the resonant (Raman) third-order nonlinearity - are affected by frequency detuning at five different temperatures with the pump wavelength fixed at $\lambda_{P}\left(v_{P}=209.205 \mathrm{THz}\right)$ and the input signal (Stokes) wavelength varied.

For each temperature, the plots of the real and imaginary parts exhibit the typical characteristics of a resonant gain medium, with the normal and anomalous dispersion regions in Fig. 5(a) being reversed compared to those of a lossy medium, and the gain curve being confined to a narrow spectral range in Fig. 5(b). These results are quite similar to the experimental results reported in Fig. 7 of [11]. When comparisons are made among the results for different temperatures, several trends immediately stand out. One, the resonance curves shift to the right with increasing temperature - by tens of GHz in each successive case - which is due to the detuning behavior shown in (4). With the pump frequency $\nu_{P}$ fixed, a decrease in the optical phonon frequency $\nu$ due to a temperature increase must be offset by a corresponding increase in the Stokes frequency $\nu_{S}$ in order to maintain the on-resonance condition, i.e., $v=v_{P}-v_{S}$. Two, such a shift is accompanied by a substantial broadening of the resonance curves, which can be attributed to a significant increase of the FWHM $2 \Gamma$, a point previously made in reference to the denominator of the Lorentzian model in (4). Finally, the peak, i.e., the on-resonance, value of the imaginary part is proportionately reduced, implying a lower gain across the entire frequency range. This is a direct consequence of the reduced Raman gain in (5) due to an increase in the FWHM.

Let us now consider the potential ramifications of the results we have just seen. Suppose a pump beam and a signal beam (Stokes component) are injected into a Raman medium with the two beams' frequencies offset by $v$, which corresponds to the optical phonon frequency at some assumed temperature, say the room temperature. If the actual temperature in the Raman medium is much higher than the assumed temperature, a likely situation in the absence of any cooling or due to a temperature gradient in the medium even with cooling, the actual gain experienced by the signal beam will be quite reduced. To see this point, observe in Figs. 5(a) and (b) that as a result of a temperature increase, we would be operating at off-resonance (e.g., operating points $A_{2}-B_{2}, A_{3}-B_{3}, A_{4}-B_{4}$ or $\left.A_{5}-B_{5}\right)$ instead of at the on-resonance $\left(A_{1}-B_{1}\right)$ condition. As will be shown in Section IIC, such changes translate into less efficient coupling between the pump and the Stokes beam, which in turn can cause a noticeable performance degradation for the Raman device being considered. 


\section{Coupled-mode equations}

In order to analyze the performance characteristics of a silicon Raman device, it is necessary to accurately account for the interaction among the pump, Stokes and anti-Stokes components as they propagate through the Raman medium. Coupled-mode approach provides a simple and yet adequate means of handling such an analysis $[19,20],[23]$. Moreover, the approach is general enough in its context so that a variety of application scenarios can be addressed with the same framework, including silicon Raman amplifiers, lasers or parametric wavelength converters [23].

In the following discussions, we shall assume that the pump, Stokes and anti-Stokes beams are all co-propagating, with suitable polarizations chosen for coupling (see [6] for example for a discussion on this). The mode profiles of the three beams are nearly identical because the three beams' wavelengths, with relatively small wavelength differences among them (pump: $1434 \mathrm{~nm}$, Stokes: $1550 \mathrm{~nm}$, anti-Stokes: $1336 \mathrm{~nm}$ ) are far removed from the fundamental mode's cutoff wavelength $(\sim 7 \mu \mathrm{m}$ due to the large index contrast between the silicon waveguide and the silica substrate). As such, we shall discard the transverse dependence of the fields and employ, without loss of any generality, a scalar treatment. By applying the slowly-varying amplitude approximation $\left(\left|d^{2} E / d z^{2}\right| \ll k \cdot|d E / d z|\right)$ to the wave equation, where $E$ is the complex amplitude of the dominant field component of the corresponding beam, and assuming $\left|E_{P}\right| \gg\left|E_{S}\right|,\left|E_{A}\right|$ the following set of coupledmode equations can be obtained [6],[13],[19,20]:

$$
\begin{aligned}
& \frac{d E_{P}}{d z}=-\frac{\left(\alpha+\alpha_{F C A}(z)\right)}{2} E_{P}-\left(\frac{\beta_{T P A} n}{2 \eta_{0}}\right)\left|E_{P}\right|^{2} E_{P} \\
& \frac{d E_{S}}{d z}=-\frac{\left(\alpha+\alpha_{F C A}(z)\right)}{2} E_{S}+\kappa_{S S} E_{S}\left|E_{P}\right|^{2}+\kappa_{S A} E_{A}^{*} E_{P}^{2} \exp (-i \Delta k z) \\
& \frac{d E_{A}^{*}}{d z}=-\frac{\left(\alpha+\alpha_{F C A}(z)\right)}{2} E_{A}^{*}+\kappa_{A A} E_{A}^{*}\left|E_{P}\right|^{2}+\kappa_{A S} E_{S} E_{P}^{* 2} \exp (+i \Delta k z)
\end{aligned}
$$

where $E_{P}, E_{S}$ and $E_{A}$ are the non-normalized complex field amplitude for the pump, Stokes and anti-Stokes beams in units of $\mathrm{V} / \mathrm{cm}$, respectively. In each of the equations above, the first term represents the combined effects of linear propagation loss and free-carrier-absorption (FCA) loss whereas the second and third terms of the last two equations involving $K$ account for the third-order nonlinear interaction among the three beams. Expressions for the coupling coefficients are given by

$$
\begin{array}{ll}
\kappa_{S S}=i\left(2 R+\frac{G}{2}+i \beta_{T P A}\right), & \kappa_{S A}=i\left(R+\frac{G}{2}\right) \\
\kappa_{A t}=-i\left(2 R-\frac{G}{2}+i \beta_{T P A}\right)^{*}, & \kappa_{A S}=-i\left(R-\frac{G}{2}\right)^{*}
\end{array}
$$

The parameters appearing in Eqs. (6) and (7) are defined as follows. $\alpha$ and $\alpha_{F C A}$ are the linear propagation and FCA loss coefficients, respectively, $\beta_{T P A}$ is the twophoton-absorption (TPA) coefficient accounting for the nonlinear loss due to the TPA process, and $\Delta k(\Delta k=$ $\left.2 k_{P}-k_{S}-k_{A}\right)$ represents the phase-mismatch involving the propagation constants of the three beams. $G$ and $R$ appearing in the coupling coefficient expressions are related to the Raman susceptibility and the third-order susceptibility accounting for non-resonant electron-photon interactions, respectively, and are defined in SI units by expressions $G=2 \pi \nu_{S} \chi_{R}^{(3)} / c n_{\text {eff, }}$, where $\chi_{R}^{(3)}$ is the appropriate Raman susceptibility $\chi_{i j m n}^{R}$ as defined previously in Eq. (4), and $R=\pi \nu_{S} \chi_{N R}^{(3)} / 2 c n_{\text {eff, }}$, where $\chi_{N R}^{(3)}$ is the non-resonant third-order susceptibility.

It must be noted that based on our earlier discussion regarding the effect of temperature on the Raman susceptibility, the value of $G$ depends on the medium's temperature as well as on the difference between the pump and Stokes component frequencies. To wit, only when the value of the shift frequency $v$ corresponding to the actual temperature is equal to the frequency difference of the injected pump and Stokes (input signal) beams, the SRS-induced gain will attain its maximum value (see Eqs. (4), (7)); for higher temperatures the gain will be reduced due to flattening and shifting of the resonance curve, the latter having the effect of frequency detuning, as explained at the end of Section IIB.

\section{RESULTS AND DISCUSSION}

In this section, we shall take the Raman wavelength converter as a specific application example of our methodology to demonstrate the type of impact that temperature effects can have on silicon Raman photonic devices. It should be pointed out, however, that the coupled-mode approach presented in the previous section is general enough to be applied towards all types of Raman devices.

Consider Fig. 6, which illustrates a wavelength converter arrangement for conversion of a C-band optical signal to an O-band optical signal. A pump beam at $1434 \mathrm{~nm}$ and a 'signal beam', i.e., the Stokes beam, at $1550 \mathrm{~nm}$ are injected into the Raman device, their frequency separation corresponding to the optical phonon frequency $15.6 \mathrm{THz}$ at $298 \mathrm{~K}$ (see Fig. 2). Due to the SRS interaction in the medium, an anti-Stokes component, i.e., a wavelength-converted signal at $1336 \mathrm{~nm}$, is generated 


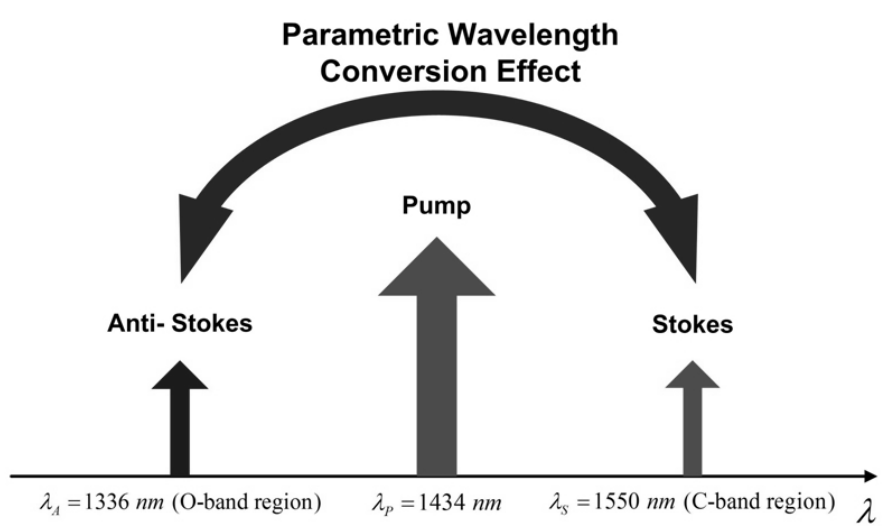

(a)

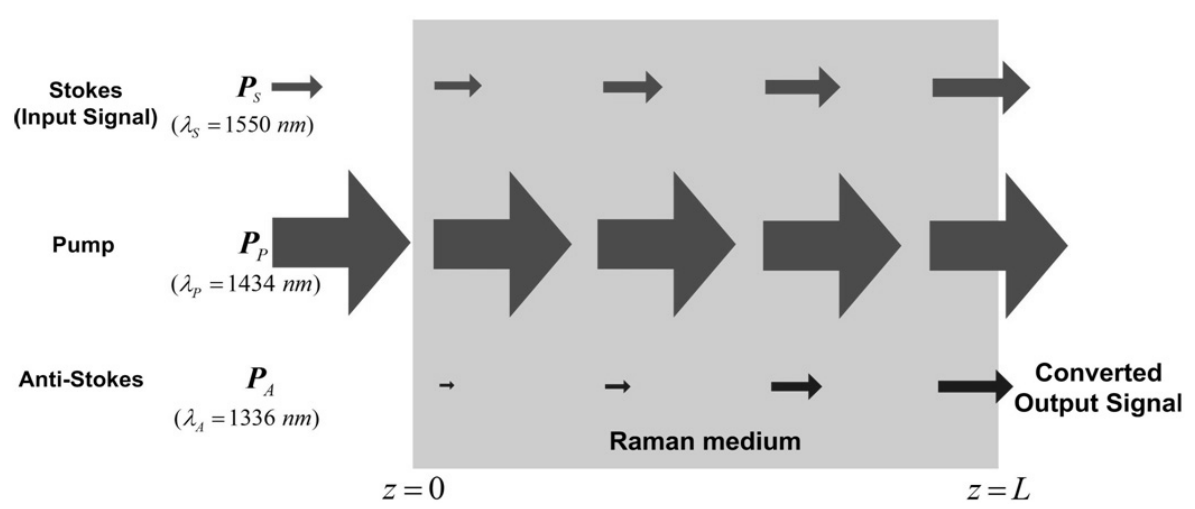

(b)

FIG. 6. Raman wavelength converter: (a) Wavelength relationship among the various components, (b) Schematic of the converter dynamics.

and emerges at the output of the device along with the pump and the Stokes beams.

The values of the material-related parameters that appeared in the coupled-mode equations earlier are assumed as follows for subsequent calculations, which are either taken directly from or chosen well in line with the values used in the references. The value of the nonresonant susceptibility $\chi_{N R}^{(3)}$ is taken as $-0.05 \times \mathrm{Im}$ $\left\{\chi_{R_{0}}^{(3)}\right\}$, where $\chi_{R_{0}}^{(3)}$ is the on-resonance Raman susceptibility at room temperature $[19,20]$, and the linear propagation loss coefficient $\alpha$ as $1 \mathrm{~dB} / \mathrm{cm}$ [24]. The TPA coefficient $\beta_{T P A}$ is assumed to be $0.5 \mathrm{~cm} / \mathrm{GW}$ [17] and the FCA loss coefficient $\alpha_{F C A}$ is calculated in unit of $\mathrm{cm}^{-1}$ as follows [4],[6]:

$$
\alpha_{F C A} \approx 6.04 \times 10^{-18} \lambda^{2}(\mu \mathrm{m}) \cdot \frac{\beta_{T P A} I_{P}^{2} \tau_{e f f}}{2 h \nu_{P}}
$$

where $I_{P}$ is the pump beam's intensity and $\tau_{\text {eff }}$ is the effective carrier lifetime, which is assumed to be $1 \mathrm{~ns}$.

Because the loss terms related to $\alpha, \beta_{T P A}, \alpha_{F C A}$ are generally much smaller than the optical nonlinearity terms containing $G$ or $R$ in the coupled-mode equations and their temperature dependences are quite complicated, we shall not take into account the temperature variation of the loss terms in our calculations. This simplification would allow us to focus solely on the temperature dependence of the Raman effect in connection with the device performance characteristics while retaining a reasonable degree of accuracy.

The coupled-mode equations given by Eq. (6), being analytically intractable in the present form, were solved numerically by using the $4^{\text {th }}$-order Runge-Kutta method, with the input pump and Stokes beam power providing the necessary boundary condition at one end.

Figs. 7(a) and (b), taken from the numerical solution obtained under the scenario described above, illustrate the evolution of the anti-Stokes and Stokes beam intensity with distance for a variety of temperature and phasematching conditions (the $\Delta k=0$ case representing perfect phase-matching) as the beams propagate through the waveguide. The input power was assumed to be 600 $\mathrm{mW}$ for the pump and $1.8 \mathrm{~mW}$ for the Stokes beam, respectively. Let us now examine the salient features of these results. First, we note that the anti-Stokes beam intensity grows from the initial value of zero (as there is no anti-Stokes beam at the input) in a quasi-linear 


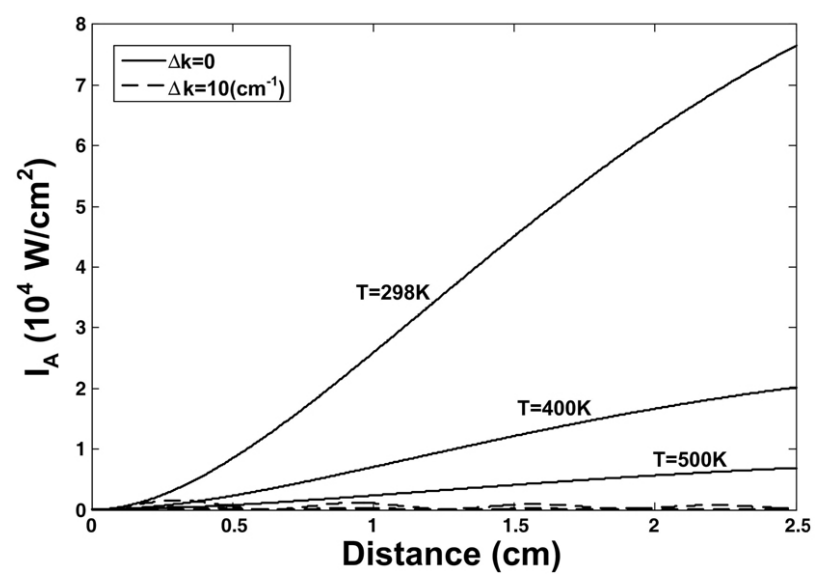

(a)

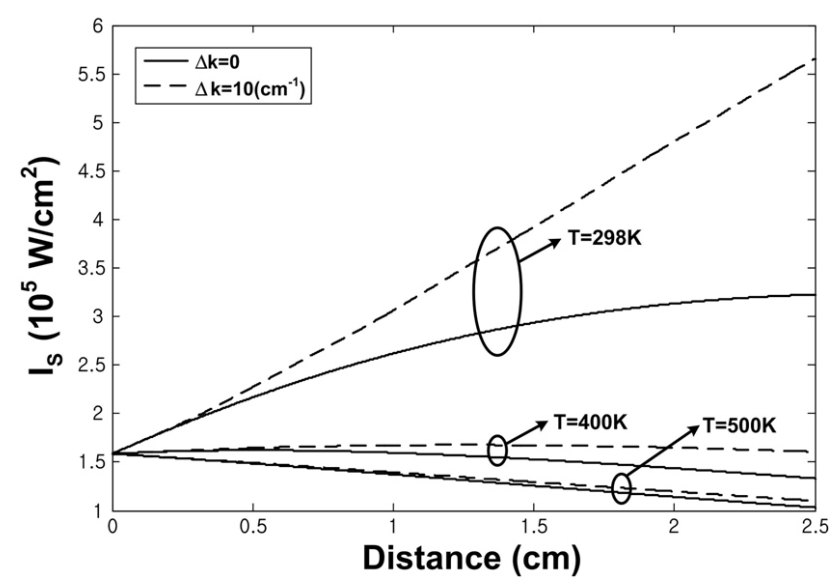

(b)

FIG. 7. Evolution of the beam intensities over distance for various temperatures and phase-matching conditions (input power: pump $=600 \mathrm{~mW}$, Stokes $=1.8 \mathrm{~mW}$ ): (a) anti-Stokes beam, (b) Stokes beam.

fashion over the distance range shown for the perfect phase-matched case of $\Delta k=0$, with the rate of increase showing a significant drop-off at higher temperatures. In fact, the anti-Stokes beam's intensity at $z=2.5 \mathrm{~cm}$ is only $\sim 0.6 \times 10^{4}$ for $\mathrm{W} / \mathrm{cm}^{2} T=500 \mathrm{~K}$ as opposed to $7.6 \times 10^{4} \mathrm{~W} / \mathrm{cm}^{2}$ for $T=298 \mathrm{~K}$, a reduction by a factor of nearly 13 ! This strong temperature dependence makes good sense given our earlier discussion on how a temperature increase reduces the nonlinear Raman gain. The quasi-linear growth behavior can be attributed to the fact that the rate of the anti-Stokes field's increase is initially governed by the last term of Eq. (6c), $\kappa_{A S} E_{S} E_{P}^{* 2}$, and is essentially independent of $E_{A}$ because $E_{A}$ starts out from zero and remains quite small. When the phase-mismatch is significant (as in the $\Delta k=10 \mathrm{~cm}^{-1}$ (ase), the anti-Stokes beam cannot sustain any growth, remaining virtually insignificant throughout propagation, with a weak quasi-periodic undulation. The reason for this behavior, which is consistent with past findings, is that an efficient transfer of power cannot take place from the pump to the anti-Stokes component due to the rapid phase oscillation $(10 \mathrm{rad} / \mathrm{cm})$ of the exponential factor $\exp (+i \Delta k z)$ in the last term of $(6 \mathrm{c})$.

The Stokes component's intensity profile contains several different features from those of the anti-Stokes component. While it is still true that a temperature increase introduces a reduction of the intensity value across the entire distance range, it is interesting to observe that the beam intensity, which starts out with some initial value, may saturate or even diminish with distance for higher temperature cases. This can be explained by the fact that at a sufficiently high temperature a power increase due to the self-dependent gain, associated with $G$ of the second term of Eq. (6b) $\kappa_{S S} E_{S}\left|E_{P}\right|^{2}$, cannot compensate enough to overcome the power losses due to the Stokes-to-anti-Stokes net power transfer and the linear absorption, TPA and FCA (see Eq. (6b)) because of the lower Raman gain. The other noteworthy feature, radically different from the anti-Stokes figure, is that the Stokes intensity is actually higher for the phase-mismatched case at all temperatures. In fact, the intensity continues to grow for the entire distance range shown for the case of $T=298 \mathrm{~K}, \Delta k=10 \mathrm{~cm}^{-1}$, although the discrepancy between the $\Delta k=0$ case and $\Delta k=10$ $\mathrm{cm}^{-1}$ case results does become increasingly small for higher temperatures. This phenomenon occurs because there is less Stokes-to-anti-Stokes power transfer when is significant due to inefficient longitudinal coupling.

We also note that for the result in Fig. 7 the output intensity for the anti-Stokes beam, i.e., $I_{A}$ at $z=2.5$ $\mathrm{cm}$, is only on the order of $10^{4} \mathrm{~W} / \mathrm{cm}^{2}$, compared to the input intensity of $1.6 \times 10^{5} \mathrm{~W} / \mathrm{cm}^{2}$ for the Stokes beam, an order of magnitude difference. Finally, we were able to verify - though supporting figures have not been included here for brevity - that the intensity distribution of the anti-Stokes component scales approximately linearly with the input Stokes power and also that the position of the maximum anti-Stokes intensity becomes increasingly closer to the input location $(z=$ 0 ) with greater input pump power.

Fig. 8 shows the Stokes-to-anti-Stokes conversion efficiency in $\mathrm{dB}$ as a function of the distance from the input location based on the result of Fig. 7(a), where the Raman parametric wavelength conversion efficiency is defined as the ratio of the anti-Stokes intensity to the input Stokes intensity $I_{S}(0)$ as follows $[6],[19,20],[23]$ :

$$
\eta=\frac{I_{A}}{I_{S}(0)}
$$

Because the conversion efficiency is essentially a normalized anti-Stokes beam intensity, physical explana- 


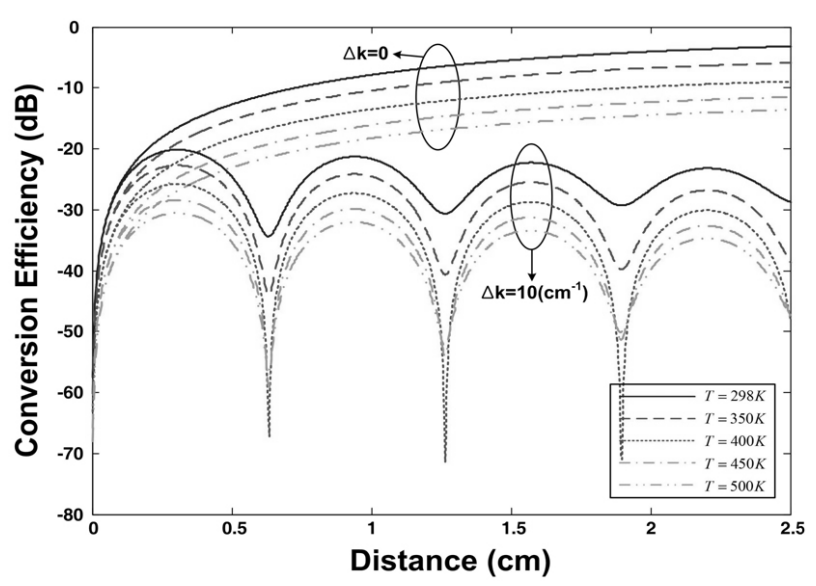

FIG. 8. Conversion efficiency as a function of distance from the input location for various temperature and phase-matching conditions (input power: pump $=600$ $\mathrm{mW}$, Stokes $=1.8 \mathrm{~mW}$.

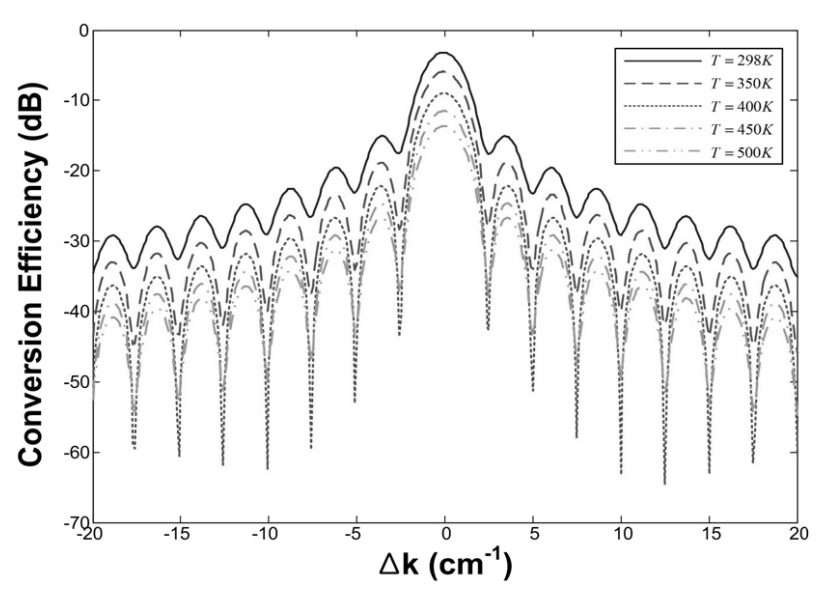

FIG. 9. Conversion efficiency as a function of phasemismatch parameter $\Delta k$ at different temperatures for device length of $2.5 \mathrm{~cm}$ (input power: pump $=600 \mathrm{~mW}$, Stokes $=1.8 \mathrm{~mW}$ ).

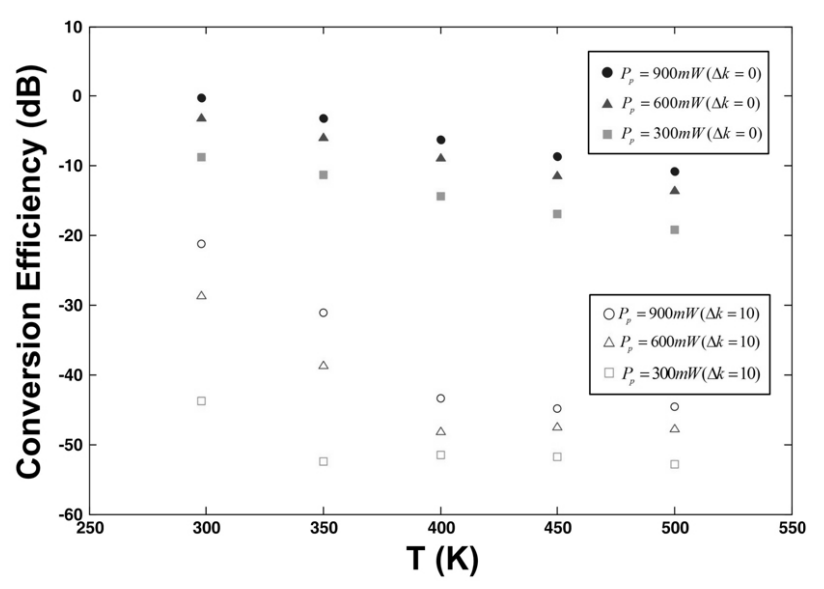

FIG. 10. Temperature dependence of the conversion efficiency with pump power and phase-mismatch as parameters. tions for its behavior with respect to temperature and phase-mismatch variations are exactly the same as with Fig. 7(a) despite the seemingly quite different appearance from the anti-Stokes intensity variations seen in Fig. 7 (a) due to the logarithmic scale. The most significant aspect about the result contained in Fig. 8 is that the maximum efficiency at the device output $(z=2.5 \mathrm{~cm})$, between -3 and $-4 \mathrm{~dB}$, occurs at $T=298 \mathrm{~K}$ with $\Delta k$ $=0$, i.e., when the temperature is the lowest and there is perfect phase-match. A temperature increase with $\Delta k$ fixed at zero leads to a rapid reduction of the conversion efficiency; in fact there is nearly a $12 \mathrm{~dB}$-drop associated with a temperature rise from 298 to $500 \mathrm{~K}$, which highlights the importance and necessity of device temperature management from a practical standpoint. There is a drop-off of similar magnitude associated with temperature increase for the $\Delta k=10 \mathrm{~cm}^{-1}$ cases but the efficiency is too low in all the cases to be of any significance.

Fig. 9 illustrates the effect that the phase-mismatch parameter $\Delta k$ has on the output conversion efficiency at several different temperatures under the assumption of $2.5 \mathrm{~cm}$ device length and the same input powers as before. The key features of the result are: (i) the maximum conversion efficiency occurs when $\Delta k=0$; (ii) the efficiency curve exhibits a Sinc $^{2}$-like behavior with respect to $\Delta k$; and lastly (iii) there is a near-uniform reduction of the efficiency across the entire $\Delta k$ range associated with a given temperature increase. These trends are consistent with our previous discussion regarding Fig. 7 (a) and also past findings on the effects of phasemismatch on parametric wavelength conversion [19],[23]. We also note that the $3-\mathrm{dB}$ width (FWHM) of the conversion efficiency is roughly equal to $2 \mathrm{~cm}^{-1}$. An important point to be made is that even though the phase-mismatch parameter was treated as an independent parameter or variable while obtaining our numerical results, careful arrangements must be made, including choosing suitable polarizations for the pump and Stokes beams, in an actual device setting in order to achieve as close to a perfect phase-matching condition as possible. This issue is quite difficult as well as complex, and interested authors should consults references such as [6,24].

Finally, Fig. 10 demonstrates the conversion efficiency's dependences on temperature for different combinations of $\Delta k$ value and the input pump power $P_{P}$. From the figure, we observe that a higher pump power unilaterally leads to a higher efficiency in all cases, which is to be expected. Other features reaffirm our earlier findings.

\section{CONCLUSION}

In this paper, we investigated the potential effects that a temperature change can have on the performances of SRS-based silicon photonic devices from a theoretical 
standpoint, a topic mostly unaddressed in the past. This issue is of paramount importance for development of field-deployable Raman silicon devices because of the high device temperature expected due to the high beam intensity and narrow crossectional area involved. We first incorporated the temperature dependences of the key SRS parameters, the resonant frequency and the FWHM linewidth of the frequency response, into the Raman gain coefficient and Raman susceptibility of silicon, which, through their role in determining the coupling coefficients, introduced temperature as an implicit variable in a simple and yet natural manner. We then solved numerically the coupled-mode equations for the example of a silicon Raman wavelength converter that generates a wavelength-converted signal at 1336 $\mathrm{nm}$ (anti-Stokes component) from the input signal of $1550 \mathrm{~nm}$ (Stokes component) to determine the temperature effects on the performance characteristics of the device.

Our results, in addition to confirming previously published theoretical and experimental results on both the silicon Raman devices in general and Raman wavelength converters, suggest that a temperature increase of $\sim 200 \mathrm{~K}$ from the room temperature can significantly reduce the conversion efficiency, by as much as $12 \mathrm{~dB}$. The primary reasons for this performance degradation were attributed to a reduction in Raman gain due to frequency-detuning effect and broadening of the SRS gain curve caused by the temperature increase. This finding suggests that, despite its attractiveness due to potential rewards from low-cost fabrication, on-chip integration capability with silicon-based optical and electronic components, and high channel-selectivity in comparison with Raman fiber devices, temperature effects must be carefully taken into account in order for silicon Raman photonic devices to become commercially feasible in optical communication applications.

\section{ACKNOWLEDGMENT}

This work was supported by 2007 Hongik University Research Fund.

\section{REFERENCES}

[1] R. Soref, "The past, present, and future of silicon photonics," J. IEEE Quantum Elec., vol. 12, no. 6, pp. 1678-1687, 2006.

[2] B. Jalali and S. Fathpour, "Silicon photonics," J. Lightwave Tech., vol. 24, no. 12, pp. 4600-4615, 2006.

[3] G. T. Reed and A. P. Knights, Silicon Photonics - An Introduction, (John Wiley \& Sons, NJ, 2004).

[4] B. Jalali, V. Raghunathan, D. Dimitropoulos, and O. Boyraz, "Raman-based silicon photonics," J. IEEE Quantum
Elec., vol. 12, no. 3, pp. 412-421, 2006.

[5] H. Rong, A. Liu, R. Jones, O. Cohen, D. Hak, R. Nicolaescu, A. Fang, and M. Paniccla, "An all-silicon Raman laser,” Nature, vol. 433, no. 20, pp. 292-294, 2005.

[6] V. Raghunathan, R. Claps, D. Dimitropoulos, and B. Jalali, "Parametric Raman wavelength conversion in scaled silicon waveguide," J. Lightwave Tech., vol. 23, no. 6, pp. 2904-2102, 2005.

[7] T. R. Hart, R. L. Aggarwal, and B. Lax, "Temperature dependence of Raman scattering in silicon," Phys. Rev., vol. 148 , no. 2 pp. 845-848, 1966.

[8] P. G. Klemens, "Anharmonic decay of optical phonons," Phys. Rev., vol. 148, no. 2 pp. 845-848, 1966.

[9] J. M. Ralston and R. K. Chang, "Spontaneous-Raman scattering efficiency and stimulated scattering in silicon," Phys. Rev. B., vol. 2, no. 6, pp. 1858-1862, 1970.

[10] M. Balkanski, R. F. Wallis, and E. Haro, "Anharmornic effects in light scattering due to optical phonon in silicon," Phys. Rev. B., vol. 28, no. 4, pp. 1928-1934, 1983.

[11] A. Compaan and H. J. Trodahl, "Resonance Raman scattering in Si at elevated temperatures," Phys. Rev. B., vol. 29, no. 2, pp. 793-801, 1984.

[12] G. P. Agrawal, Nonlinear Fiber Optics, $3^{\text {rd }}$ ed., (Academic Press, San Diego, 2002).

[13] E. Golovchenko, P. V. Mamyshev, A. N. Pilipetskii, and E. M. Dianov, "Mutual influence of parametric effects and stimulated Raman scattering in optical waveguide," J. IEEE Quantum Elec., vol. 26, no. 10, pp. 1815-1820, 1990.

[14] R. Loudon, "The Raman effect in crystals," Advan. Phys. vol. 13, pp. 423-482, 1964.

[15] C. Kittel, Introduction to Solid State Physics, $8^{\text {th }}$ ed., (John Wiley \& Sons, Hoboken, NJ, 2005).

[16] P. Y. Yu and M. Cardona, Fundamentals of Semiconductors, $3^{\text {rd }}$ ed., (Springer-Verlag, Berlin, 2001).

[17] H. Rong, A. Liu, R. Nicolaescu, M. Paniccia, O. Cohen, and D. Hak, "Raman gain and nonlinear optical absorption measurements in a low-loss silicon waveguide," Appl. Phys. Lett., vol. 85, no. 12, pp. 2196-2198, 2004.

[18] J. Niu, J. Sha, and D. Yang, "Temperature dependence of first-order Raman scattering in silicon nanowire," Scrip. Mat., vol. 55, pp. 183-186, 2006.

[19] Y. R. Shen and N. Bloembergen, "Theory of stimulated Brillouin and Raman scattering," Phys. Rev. vol. 137, no. 6A, pp. A1787-A1805, 1965.

[20] Y. R. Shen, The Principles of Nonlinear Optics, (John Wiley \& Sons, New York, 1984).

[21] R. Claps and D. Dimitropoulos, Y. Han, B. Jalali, "Observation of Raman emission in silicon waveguide at 1.54um," Opt. Exp., vol. 10. no. 22, pp. 1305- 1313, 2002 .

[22] J. Touminen, T. Niemi, and H. Ludvigsen, "Wavelength reference for optical telecommunications based on a temperature-tunable silicon etalon," Rev. Sci. Instrum., vol. 74, no. 8, pp. 3620-3623, 2003.

[23] R. W. Boyd, Nonlinear Optics, $2^{\text {nd }}$ ed., (Academic Press, San Diego, 2003).

[24] L. Pavesi and D. J. Lockwood, Silicon Photonics, (Springer-Verlag, Berlin, 2004). 